Bull. Korean Math. Soc. 52 (2015), No. 2, pp. 409-419

http://dx.doi.org/10.4134/BKMS.2015.52.2.409

\title{
CAYLEY-SYMMETRIC SEMIGROUPS
}

\author{
YONGWEN ZHU
}

\begin{abstract}
The concept of Cayley-symmetric semigroups is introduced, and several equivalent conditions of a Cayley-symmetric semigroup are given so that an open problem proposed by Zhu [19] is resolved generally. Furthermore, it is proved that a strong semilattice of self-decomposable semigroups $S_{\alpha}$ is Cayley-symmetric if and only if each $S_{\alpha}$ is Cayleysymmetric. This enables us to present more Cayley-symmetric semigroups, which would be non-regular. This result extends the main result of Wang [14], which stated that a regular semigroup is Cayleysymmetric if and only if it is a Clifford semigroup. In addition, we discuss Cayley-symmetry of Rees matrix semigroups over a semigroup or over a 0-semigroup.
\end{abstract}

\section{Introduction}

Many research papers were devoted to Cayley graphs of semigroups (see, for example, $[1,3,4,5,6,7,8,9,10,11,12,13,16,17])$. Based on these works, the author first introduced the concept of generalized Cayley graphs of semigroups in [18], where some fundamental properties of generalized Cayley graphs of semigroups were studied. This work was extended in [19], where various combinatorial issues relating to generalized Cayley graphs were addressed. Especially, in Remark 3.8 of [19], the author proposed the following question: It may be interesting to characterize semigroups $S$ such that $\operatorname{Cay}\left(S, S_{l}\right)=\operatorname{Cay}\left(S, S_{r}\right)$, where $S_{l}$ and $S_{r}$ denote the left and right universal relations on $S^{1}$ respectively. This problem was partially solved by Wang in [14], where it was proved that for any regular semigroup $S, \operatorname{Cay}\left(S, S_{l}\right)=\operatorname{Cay}\left(S, S_{r}\right)$ if and only if $S$ is a Clifford semigroup.

Following $[14,18,19]$, we continue to study the generalized Cayley graphs of semigroups in the present paper. Based on the problem mentioned as above, we introduce naturally the concept of Cayley-symmetric semigroups, in view of which the above problem may be restated as follows: When is a semigroup

Received August 28, 2013; Revised November 10, 2013.

2010 Mathematics Subject Classification. 05C25, 20M12, $20 \mathrm{M} 17$.

Key words and phrases. generalized Cayley graph, Cayley-symmetric semigroup, strong semilattice of semigroups, self-decomposable. 
Cayley-symmetric? Consequently, the main result of Wang [14] would be restated as follows: A regular semigroup is Cayley-symmetric if and only if it is a Clifford semigroup. As the most general answers to the above problem, several equivalent conditions of a Cayley-symmetric semigroup are given in this paper. We also generalize the notion of Clifford semigroups and establish a necessary and sufficient condition for a semilattices of semigroups to be Cayleysymmetric. Consequently, we can display some Cayley-symmetric semigroups which are not regular. In this sense, our results also extend the main result of [14]. In addition, the Cayley-symmetry of Rees matrix semigroups over a semigroup or over a 0 -semigroup is also discussed so that the corresponding equivalent conditions are given.

\section{Preliminaries}

Recall that if $S$ is an ideal of a semigroup $T$, then we call $T$ an ideal extension of $S$. Let $T^{1}$ be the semigroup $T$ with an identity adjoined if necessary.

Definition 2.1 ([18, 19]). Let $T$ be an ideal extension of a semigroup $S$ and $\rho \subseteq T^{1} \times T^{1}$. The Cayley graph $\operatorname{Cay}(S, \rho)$ of $S$ relative to $\rho$ is defined as the graph with vertex set $S$ and edge set $E(\operatorname{Cay}(S, \rho))$ consisting of those ordered pairs $(a, b)$, where $x a y=b$ for some $(x, y) \in \rho$. We also call the Cayley graphs defined in this way the generalized Cayley graphs, in order to distinguish them from the usual ones.

Assume that $S$ is a semigroup and $a \in S$. Then $J(a)=S^{1} a S^{1}\left(L(a)=S^{1} a\right.$, $R(a)=a S^{1}$ ) is the principal (left, right) ideal generated by $a$ (cf. $[2,20]$ ). Let $S_{l}=S^{1} \times\{1\}$ (the left universal relation on $S^{1}$ ), $S_{r}=\{1\} \times S^{1}$ (the right universal relation on $S^{1}$ ) and $\omega_{S}=S^{1} \times S^{1}$ (the universal relation on $S^{1}$ ). Then the generalized Cayley graphs $\operatorname{Cay}\left(S, S_{l}\right), \operatorname{Cay}\left(S, S_{r}\right)$ and $\operatorname{Cay}\left(S, S_{\omega}\right)$ are called the left universal, right universal and universal Cayley graphs of $S$, respectively.

As mentioned in [19, Remark 3.8], it would be interesting to characterize semigroups $S$ such that $\operatorname{Cay}\left(S, S_{l}\right)=\operatorname{Cay}\left(S, S_{r}\right)$. We shall answer this question in general in this paper. It would be convenient to give the following definition.

Definition 2.2. A semigroup $S$ is called Cayley-symmetric if $\operatorname{Cay}\left(S, S_{l}\right)=$ $\operatorname{Cay}\left(S, S_{r}\right)$.

Assume that $T$ is an ideal extension of a semigroup $S$. Then the generalized Cayley graphs $\mathrm{Cay}\left(S, T_{l}\right), \mathrm{Cay}\left(S, T_{r}\right)$ and $\mathrm{Cay}\left(S, T_{\omega}\right)$ are called the left $T$ universal, right $T$-universal and $T$-universal Cayley graphs of $S$, respectively.

We also consider a more general symmetry problem in this paper. As a natural generalization of Definition 2.2, we have the following definition.

Definition 2.3. Let $T$ be an ideal extension of a semigroup $S$. If Cay $\left(S, T_{l}\right)=$ $\operatorname{Cay}\left(S, T_{r}\right)$, then we say that $S$ is Cayley-symmetric in $T$.

Throughout the paper, graphs are directed graphs without multiple edges, but possibly with loops, or digraphs in terms of $[1,11]$. As in $[18,19]$, we always 
equate two graphs isomorphic to each other if no confusion occurs. For a graph $\Gamma$, denote by $V(\Gamma)$ and $E(\Gamma)$ its vertex set and edge set, respectively. A graph $\Gamma_{0}$ is called a subgraph of a graph $\Gamma$ if $V\left(\Gamma_{0}\right) \subseteq V(\Gamma)$ and $E\left(\Gamma_{0}\right) \subseteq E(\Gamma)$. A graph $\Gamma_{0}$ is called an induced subgraph of a graph $\Gamma$ if $\Gamma_{0}$ is a subgraph of $\Gamma$ and the following condition is satisfied: for any $a, b \in V\left(\Gamma_{0}\right),(a, b) \in E\left(\Gamma_{0}\right)$ if and only if $(a, b) \in E(\Gamma)$. Any book on graph theory, e.g., [4, 15], will provide terminology which may be used in this paper without definition.

For notions and notations of semigroup theory, we refer the reader to [2].

\section{Cayley-symmetric semigroups}

In this section, we answer the following question in general: When is a semigroup Cayley-symmetric? More generally, we answer the following question: When is a semigroup Cayley-symmetric in its a given ideal extension?

Assume that $T$ is an ideal extension of a semigroup $S$ and that $A$ is a subset of $S$. Then the ideal, left ideal and right ideal generated by $A$ in $T$ are denoted by $J_{T}(A), L_{T}(A)$ and $R_{T}(A)$, respectively. If $A=\{a\}$, then instead of writing $J_{T}(A), L_{T}(A)$ and $R_{T}(A)$, we write $J_{T}(a), L_{T}(a)$ and $R_{T}(a)$, which are called the ideal, left ideal and right ideal generated by a in $T$, respectively. In the case that $S=T$, we write $J(A), L(A), R(A), J(a), L(a)$ and $R(a)$ instead of writing $J_{T}(A), L_{T}(A), R_{T}(A), J_{T}(a), L_{T}(a)$ and $R_{T}(a)$, respectively. It is clear that $J_{T}(a)=T^{1} a T^{1}, L_{T}(a)=T^{1} a$ and $R_{T}(a)=a T^{1}$. Thus $J(a)=S^{1} a S^{1}$, $L(a)=S^{1} a$ and $R(a)=a S^{1}$ (cf. [2]). It is easy to show the following lemma.

Lemma 3.1. Let $T$ be an ideal extension of a semigroup $S$ and $A$ a subset of $S$. Then $J_{T}(A)=\bigcup_{a \in A} J_{T}(a), L_{T}(A)=\bigcup_{a \in A} L_{T}(a)$, and $R_{T}(A)=\bigcup_{a \in A} R_{T}(a)$.

Furthermore, we can prove the next lemma.

Lemma 3.2. If $T$ is an ideal extension of a semigroup $S$, then the following statements are equivalent:

(1) $L_{T}(a)=R_{T}($ a) for every $a \in S$;

(2) $L_{T}(A)=R_{T}(A)$ for every $A \subseteq S$;

(3) $L_{T}(a)$ is a right ideal of $T$ and $R_{T}(a)$ is a left ideal of $T$ for every $a \in S$;

(4) $L_{T}(A)$ is a right ideal of $T$ and $R_{T}(A)$ is a left ideal of $T$ for every $A \subseteq S$.

Proof. $(1) \Rightarrow(2)$ : In view of Lemma 3.1, we have $L_{T}(A)=\bigcup_{a \in A} L_{T}(a)$ and $R_{T}(A)=\bigcup_{a \in A} R_{T}(a)$, which together with the condition (1) implies that

$$
L_{T}(A)=\bigcup_{a \in A} L_{T}(a)=\bigcup_{a \in A} R_{T}(a)=R_{T}(A) .
$$

$(2) \Rightarrow(1)$ : This is trivial.

$(1) \Rightarrow(3)$ : This is obvious since $L_{T}(a)$ is a left ideal and $R_{T}(a)$ is a right ideal of $T$. 
$(3) \Rightarrow(1)$ : Note that $L_{T}(a) \subseteq J_{T}(a)$ by definitions. Since $L_{T}(a)$ is a right ideal of $T, J_{T}(a)=T^{1} a T^{1}=L_{T}(a) T^{1} \subseteq L_{T}(a) \subseteq J_{T}(a)$. Hence $L_{T}(a)=$ $J_{T}(a)$. A similar argument shows that $R_{T}(a)=J_{T}(a)$. So $L_{T}(a)=J_{T}(a)=$ $R_{T}(a)$.

$(4) \Rightarrow(3)$ : This is trivial.

$(2) \Rightarrow(4)$ : This is obvious since $L_{T}(A)$ is a left ideal and $R_{T}(A)$ is a right ideal of $T$.

As the main result of this section, the following theorem characterizes the semigroups that are Cayley-symmetric in their ideal extensions.

Theorem 3.3. If $T$ is an ideal extension of a semigroup $S$, then the following statements are equivalent:

(1) $S$ is Cayley-symmetric in $T$;

(2) $L_{T}(a)=R_{T}(a)$ for every $a \in S$;

(3) $L_{T}(A)=R_{T}(A)$ for every $A \subseteq S$;

(4) $L_{T}(a)$ is a right ideal of $T$ and $R_{T}(a)$ is a left ideal of $T$ for every $a \in S$;

(5) $L_{T}(A)$ is a right ideal of $T$ and $R_{T}(A)$ is a left ideal of $T$ for every $A \subseteq S$.

Proof. In light of Lemma 3.2, we only need to prove the equivalence of (1) and (2). According to the definition of generalized Cayley graphs, we know that

$$
\begin{aligned}
& (a, b) \in E\left(\operatorname{Cay}\left(S, T_{l}\right)\right) \\
\Leftrightarrow \quad & b=x a \text { for some } \quad x \in T^{1} \\
\Leftrightarrow \quad & b \in T^{1} a=L_{T}(a)
\end{aligned}
$$

and that

$$
\begin{aligned}
& (a, b) \in E\left(\operatorname{Cay}\left(S, T_{r}\right)\right) \\
\Leftrightarrow & b=a y \text { for some } y \in T^{1} \\
\Leftrightarrow & b \in a T^{1}=R_{T}(a) .
\end{aligned}
$$

If $\operatorname{Cay}\left(S, T_{l}\right)=\operatorname{Cay}\left(S, T_{r}\right)$, then for any $a, b \in S,(a, b) \in E\left(\operatorname{Cay}\left(S, T_{l}\right)\right)$ if and only if $(a, b) \in E\left(\operatorname{Cay}\left(S, T_{r}\right)\right)$. Thus for all $a, b \in S$ we have that $b \in L_{T}(a)$ if and only if $b \in R_{T}(a)$. Since $S$ is an ideal of $T, L_{T}(a)=T^{1} a \subseteq S$ and $R_{T}(a)=a T^{1} \subseteq S$ for every $a \in S$. So $L_{T}(a)=R_{T}(a)$ for every $a \in S$.

Conversely, suppose that $L_{T}(a)=R_{T}(a)$ for every $a \in S$. Then for all $a, b \in$ $S$ we have that $b \in L_{T}(a)$ if and only if $b \in R_{T}(a)$. Hence $(a, b) \in E\left(\operatorname{Cay}\left(S, T_{l}\right)\right)$ if and only if $(a, b) \in E\left(\operatorname{Cay}\left(S, T_{r}\right)\right)$. That is to say that $\operatorname{Cay}\left(S, T_{l}\right)=\operatorname{Cay}\left(S, T_{r}\right)$.

Using Theorem 3.3 with $T=S$, we immediately have the following corollary, which answers the open question raised by the author in [19].

Corollary 3.4. For any semigroup $S$, the following statements are equivalent:

(1) $S$ is Cayley-symmetric;

(2) $L(a)=R(a)$ for every $a \in S$; 
(3) $L(A)=R(A)$ for every $A \subseteq S$;

(4) $L(a)$ is a right ideal and $R(a)$ is a left ideal for every $a \in S$;

(5) $L(A)$ is a right ideal and $R(A)$ is a left ideal for every $A \subseteq S$;

(6) Every left ideal is a right ideal and every right ideal is a left ideal.

In the remainder of this section, we present some Cayley-symmetric semigroups.

Suppose that $S$ is a semigroup with zero 0 and that $S=\bigcup_{\alpha \in I} S_{\alpha}$, where each $S_{\alpha}$ is a subsemigroup of $S$, and where

$$
S_{i} S_{j}=S_{i} \cap S_{j}=\{0\} .
$$

Then we call $S$ is a 0-direct union of $S_{\alpha}$ 's ([2]). If we further suppose that every $S_{\alpha}$ is Cayley-symmetric, then $S_{\alpha}^{1} a=a S_{\alpha}^{1}$ for all $a \in S_{\alpha}$ by Corollary 3.4. It follows that $S^{1} a=a S^{1}$ for all $a \in S$. Hence $S$ is Cayley-symmetric by Corollary 3.4 again. Consequently, we obtain the following corollary.

Corollary 3.5. Assume that $S$ is a 0 -direct union of semigroups $S_{\alpha}$ 's. If every $S_{\alpha}$ is Cayley-symmetric, then so is $S$.

If $G$ is a group, then we have $a G=G=G a$ for every $a \in G$, which means that $G$ is Cayley-symmetric by Corollary 3.4. Let $G^{0}=G \cup\{0\}$, where 0 is an adjoined zero. Then we call $G^{0}$ a 0-group. Also we have $a G^{0}=G^{0} a$ for every $a \in G^{0}$, which means that $G^{0}$ is also Cayley-symmetric by Corollary 3.4 again. As a direct consequence of Corollary 3.5, we have the following corollary.

Corollary 3.6. If $S$ is a 0 -direct union of some 0-groups, then $S$ is Cayleysymmetric.

\section{Cayley-symmetry of Rees matrix semigroups}

This section is devoted to the Cayley-symmetry of Rees matrix semigroups so that a necessary and sufficient condition is given for a Rees matrix semigroup to be Cayley-symmetric. The main results of this section are Theorems 4.4 and 4.7 .

Let $S$ be a semigroup, let $I, \Lambda$ be nonempty sets and let $P=\left(p_{\lambda i}\right)$ be a $\Lambda \times I$ matrix with entries in $S^{1}$. (Note that here is $S^{1}$ rather than $S$.) Let $T=I \times S \times \Lambda$, and define a multiplication on $T$ by

$$
(i, a, \lambda)(j, b, \mu)=\left(i, a p_{\lambda j} b, \mu\right) .
$$

Then $T$ is a semigroup, which is called the $I \times \Lambda$ Rees matrix semigroup over the semigroup $S$ with the sandwich matrix $P$ and denoted by $\mathcal{M}[S ; I, \Lambda ; P]$. Recall that a semigroup is called completely simple if it is simple and if it contains a primitive idempotent. By [2, Theorem 3.3.1], a semigroup is completely simple if and only if it is isomorphic to a Rees matrix semigroup $\mathcal{M}[G ; I, \Lambda ; P]$ over a group $G$.

To study the Cayley-symmetry of a Rees matrix semigroup over a semigroup, we need some new terminologies. As a generalization of the identity of a semigroup, a mid-identity of a semigroup is defined as follows. 
Definition 4.1. An element $u$ of a semigroup $S$ is called a mid-identity, if for all $x, y \in S, x u y=x y$.

The next terms generalize the concept of invertible elements of a group.

Definition 4.2. Let $S$ be a semigroup and $p \in S$. If there exists $q \in S$ such that $p q(q p)$ is a mid-identity, then $p$ is called left (right) factor of a mididentity. If $p$ is not only a left factor of a mid-identity but also a right factor of a mid-identity, then $p$ is called mid-invertible.

It is clear that the identity of a semigroup is a mid-identity and it is also mid-invertible. Note that if $S$ is a semigroup with an identity 1 and with a mid-identity $u$, then $1 u 1=11$ which means that $u=1$. A non-trivial example is as follows.

Example 4.3. Let $S=\langle p, q\rangle$ be the matrix semigroup (under the usual multiplication of matrices) generated by two matrices $p=\left(\begin{array}{lll}2 & 0 & 0 \\ 0 & 0 & 1 \\ 0 & 0 & 0\end{array}\right)$ and $q=$ $\left(\begin{array}{ccc}2^{-1} & 0 & 0 \\ 0 & 0 & 1 \\ 0 & 0 & 0\end{array}\right)$. Then $u=p q=q p=\left(\begin{array}{lll}1 & 0 & 0 \\ 0 & 0 & 0 \\ 0 & 0 & 0\end{array}\right)$ is a mid-identity rather than an identity of $S$. Furthermore, both $p$ and $q$ are mid-invertible.

As one of two main results of this section, the next theorem gives an equivalent condition for a Rees matrix semigroup over a semigroup to be Cayleysymmetric.

Theorem 4.4. Let $T=\mathcal{M}[S ; I, \Lambda ; P]$ be an $I \times \Lambda$ Rees matrix semigroup over a semigroup $S$, where the sandwich matrix $P=\left(p_{\lambda i}\right)$ is a $\Lambda \times I$ matrix with entries in $S^{1}$. Assume that $p_{11}=p$ is a mid-invertible element of $S^{1}$. Then $T$ is Cayley-symmetric if and only if $|I|=|\Lambda|=1$ and $S$ is Cayley-symmetric.

Proof. Necessity. Suppose that $T$ is Cayley-symmetric. By (4.1), one can deduce that for any $i \in I, \lambda \in \Lambda, a \in S$,

$$
\begin{aligned}
& \left.(i, a, \lambda) T^{1}=\left\{\left(i, a p_{\lambda j} b, \mu\right) \mid j \in I, \mu \in \Lambda, b \in S\right)\right\}, \\
& \left.T^{1}(i, a, \lambda)=\left\{\left(j, b p_{\mu i} a, \lambda\right) \mid j \in I, \mu \in \Lambda, b \in S\right)\right\} .
\end{aligned}
$$

Since $T$ is Cayley-symmetric, we have

$$
(i, a, \lambda) T^{1}=T^{1}(i, a, \lambda)
$$

by Corollary 3.4. From (4.2), (4.3) and (4.4), it follows that $|I|=|\Lambda|=1$. Thus we may set $I=\{1\}$ and $\Lambda=\{1\}$. Then we have that

$$
\{(1, a, 1),(1, a p b, 1) \mid b \in S\}=\{(1, a, 1),(1, c p a, 1) \mid c \in S\}
$$

holds for all $a \in S$. It follows that for every $a \in S$,

$$
\{a, a p b \mid b \in S\}=\{a, c p a \mid c \in S\} .
$$


By the assumption of the theorem, we can suppose that there exist $u, v, q, r \in S$ such that $u, v$ are mid-identity, and that $p q=u$ and $r p=v$. Thus for any $a, b \in S$, according to (4.6), we have that

$$
\begin{aligned}
a b & =a u b \\
& =a p q b \\
& =\left\{\begin{array}{l}
a \\
c p a .
\end{array}\right.
\end{aligned}
$$

This shows that $a S^{1} \subseteq S^{1} a$. A symmetric argument shows that $S^{1} a \subseteq a S^{1}$. Hence $a S^{1}=S^{1} a$. So we have that $S$ is Cayley-symmetric by Corollary 3.4.

Sufficiency. Assume that $I=\{1\}$ and $\Lambda=\{1\}$ and that $S$ is Cayleysymmetric. To show that $T$ is Cayley-symmetric, it only remains to prove that (4.6) holds. For this, let $a, b \in S$. By Corollary 3.4 again, we obtain that

$$
\{a, a b \mid b \in S\}=\{a, c a \mid c \in S\} .
$$

Then $a p b=a$ or $a p b=c a$ (for some $c \in S$ ). If the case is the former, then it is easy to see that

$$
\{a, a p b \mid b \in S\} \subseteq\{a, c p a \mid c \in S\} .
$$

If it is the latter case, i.e., if there exists $c \in S$ such that $a p b=c a$, then we have that

$$
a p b=c a=c v a=a r p a,
$$

which shows that (4.8) remains true. By a symmetric argument, we can prove the inverse conclusion of (4.8). Therefore, (4.6) holds. This completes the proof.

Note that each element of a group is mid-invertible. So we have the following corollary immediately.

Corollary 4.5. A completely simple semigroup is Cayley-symmetric if and only it is isomorphic to a group.

Observe that if $p_{\lambda i}=1$ for all $i \in I$ and $\lambda \in \Lambda$, then $T=\mathcal{M}[S ; I, \Lambda ; P]$ is isomorphic to the direct product of $S$ and the rectangular band $B=I \times \Lambda$. Consequently, we obtain the following corollary.

Corollary 4.6. A direct product of a semigroup $S$ and a rectangular band $B$ is Cayley-symmetric if and only if $|B|=1$ and $S$ is Cayley-symmetric.

Let us turn our attention to the Cayley-symmetry of a Rees matrix semigroup over a 0 -semigroup $S^{0}\left(S^{0}=S \cup\{0\}\right.$ where 0 is an adjoined zero).

Assume that $S$ is a semigroup. Let $I, \Lambda$ be nonempty sets and let $P=\left(p_{\lambda i}\right)$ be a $\Lambda \times I$ matrix with entries in $S^{1,0}\left(S^{1,0}=S^{1} \cup\{0\}\right)$. Suppose that $P$ is regular, in the sense that no row or column of $P$ consists entirely of zeros. Formally,

$$
\begin{aligned}
& (\forall i \in I)(\exists \lambda \in \Lambda) p_{\lambda i} \neq 0, \\
& (\forall \lambda \in \Lambda)(\exists i \in I) p_{\lambda i} \neq 0 .
\end{aligned}
$$


Let $T^{0}=(I \times S \times \Lambda) \cup\{0\}$, and define a composition on $T^{0}$ by

$$
(i, a, \lambda)(j, b, \mu)= \begin{cases}\left(i, a p_{\lambda j} b, \mu\right), & \text { if } p_{\lambda j} \neq 0 \\ 0, & \text { if } p_{\lambda j}=0\end{cases}
$$

Then $T^{0}$ is a semigroup, which is called the $I \times \Lambda$ Rees matrix semigroup over the 0-semigroup $S^{0}$ with the regular sandwich matrix $P$ and denoted by $\mathcal{M}^{0}[S ; I, \Lambda ; P]$. Recall that a semigroup is called completely 0 -simple if it is 0 -simple and if it contains a primitive idempotent. By Rees Theorem (see [2, Theorem 3.2.3]), a semigroup is completely 0-simple if and only if it is isomorphic to a Rees matrix semigroup $\mathcal{M}^{0}[G ; I, \Lambda ; P]$ over a 0 -group $G^{0}$.

As the other main result of this section, the next theorem gives an equivalent condition of a Cayley-symmetric Rees matrix semigroup over a 0-semigroup.

Theorem 4.7. Let $T^{0}=\mathcal{M}^{0}[S ; I, \Lambda ; P]$ be an $I \times \Lambda$ Rees matrix semigroup over a 0 -semigroup $S^{0}$, where the sandwich matrix $P=\left(p_{\lambda i}\right)$ is a regular $\Lambda \times I$ matrix with entries in $S^{1,0}$. Assume that $p_{11}=p$ is a mid-invertible element of $S^{1,0}$. Then $T^{0}$ is Cayley-symmetric if and only if $|I|=|\Lambda|=1$ and $S$ is Cayley-symmetric.

Proof. It is analogous to the proof of Theorem 4.4 .

As a direct consequence of Theorem 4.7, we have the following corollary.

Corollary 4.8. A completely 0-simple semigroup is Cayley-symmetric if and only it is isomorphic to a 0-group.

\section{Cayley-symmetry of strong semilattices of semigroups}

In this section, we shall introduce the concept of strong semilattices of semigroups, which is a natural generalization of the notion of Clifford semigroups. The main result of this section is Theorem 5.3, which gives a necessary and sufficient condition for a strong semilattice of semigroups to be Cayley-symmetric. Consequently, we can display more Cayley-symmetric semigroups which would not be regular. This means that our construction will provide a more universal class of Cayley-symmetric semigroups than the Clifford semigroups. In this sense, our result generalizes that of [14], which stated that a regular semigroup is Cayley-symmetric if and only if it is a Clifford semigroup.

Suppose that we have a semilattice $Y$ and a set of semigroups $S_{\alpha}$ indexed by $Y$, and suppose that, for all $\alpha \geq \beta$ in $Y$ there exists a morphism $\phi_{\alpha, \beta}: S_{\alpha} \rightarrow$ $S_{\beta}$ such that: (1) for each $\alpha \in Y, \phi_{\alpha, \alpha}=1_{S_{\alpha}}$, the identity mapping on $S_{\alpha} ;(2)$ $\phi_{\alpha, \beta} \phi_{\beta, \gamma}=\phi_{\alpha, \gamma}$ for all $\alpha, \beta, \gamma \in Y$ such that $\alpha \geq \beta \geq \gamma$. Let $S=\dot{\bigcup}_{\alpha \in Y} S_{\alpha}$, the disjoint union of $S_{\alpha}$ 's. Define a multiplication on $S$ by the rule that, for each $a \in S_{\alpha}$ and $b \in S_{\beta}$,

$$
a b=(a) \phi_{\alpha, \alpha \beta}(b) \phi_{\beta, \alpha \beta} .
$$


Then $S$ is a semigroup, called the strong semilattice of semigroups $S_{\alpha}$ (cf. [18, Definition 3.11]). We write

$$
S=\mathcal{S}\left[Y ; S_{\alpha} ; \phi_{\alpha, \beta}\right]
$$

To study Cayley-symmetry of a strong semilattice of semigroups, we need a new term, so called self-decomposable semigroups, which is also a generalization of the notion of monoids or that of regular semigroups.

Definition 5.1. Let $S$ be a semigroup. If for every $a \in S, a \in S a \cap a S$, then we call $S$ self-decomposable.

Note that if $S$ is a self-decomposable semigroup, then $L(a)=S^{1} a=S a$ and $R(a)=a S^{1}=a S$. Thus according to Corollary 3.4, we immediately obtain the following lemma, which provides a general answer to that problem in [19] for the self-decomposable semigroup class and will be used repeatedly later.

Lemma 5.2. Let $S$ be a self-decomposable semigroup. Then $S$ is Cayleysymmetric if and only if $S a=a S$ for all $a \in S$.

As our main result of this section, the next theorem gives a necessary and sufficient condition for a strong semilattice of self-decomposable semigroups to be Cayley-symmetric.

Theorem 5.3. Suppose that $S=\mathcal{S}\left[Y ; S_{\alpha} ; \phi_{\alpha, \beta}\right]$, where each $S_{\alpha}$ is self-decomposable. Then $S$ is Cayley-symmetric if and only if for every $\alpha \in Y, S_{\alpha}$ is Cayley-symmetric.

Proof. Observe that $S=\mathcal{S}\left[Y ; S_{\alpha} ; \phi_{\alpha, \beta}\right]$ is self-decomposable since for every $\alpha$, $S_{\alpha}$ is self-decomposable.

Necessity. Assume that $S$ is Cayley-symmetric. Let $a, b \in S_{\alpha}$ with $\alpha \in Y$. According to Lemma 5.2, there exist $\beta \in Y$ and $c \in S_{\beta}$ such that $a b=c a$. It is clear that $\beta \geq \alpha$ since $a b \in S_{\alpha}$. Since $S_{\alpha}$ is self-decomposable, there exists $u \in S_{\alpha}$ such that $a=u a$. It follows that $a b=c a=c(u a)=(c u) a$, where $c u \in S_{\alpha}$. We have proved that $a S_{\alpha} \subseteq S_{\alpha} a$. Similarly, we have that $S_{\alpha} a \subseteq a S_{\alpha}$, which means that $S_{\alpha} a=a S_{\alpha}$. Thus by Lemma 5.2 again, $S_{\alpha}$ is Cayley-symmetric.

Sufficiency. Suppose that for all $\alpha, S_{\alpha}$ is Cayley-symmetric. Let $a \in S_{\alpha}$ and $b \in S_{\beta}$ with $\alpha, \beta \in Y$. Set $\alpha \beta=\gamma \in Y$, then $\alpha, \beta \geq \gamma$. Since $a b \in S_{\gamma}$ and $S_{\gamma}$ is self-decomposable, there exist $x, y \in S_{\gamma}$ such that $a b=x(a b)=(a b) y$. It follows that $a b=x(a b) y=(x a)(b y)$, where $x a, b y \in S_{\gamma}$. Since $S_{\gamma}$ is Cayleysymmetric, from Lemma 5.2 we deduce that there exists $z \in S_{\gamma}$ such that $(x a)(b y)=z(x a)=(z x) a$, which implies that $a b=(z x) a \in S a$. We have proved that $a S \subseteq S a$. The inverse conclusion may be proved in a similar way. Hence $a S=S a$. Therefore, by Lemma 5.2 again, $S$ is Cayley-symmetric. This completes the proof.

Now suppose that $S$ is a Clifford semigroup. According to [2, Theorem 4.1], $S=\mathcal{S}\left[Y ; G_{\alpha} ; \phi_{\alpha, \beta}\right]$, a strong semilattice of groups $G_{\alpha}$ 's. Of course, every group 
$G_{\alpha}$ is Cayley-symmetric. Thus using Theorem 5.3, we immediately obtain the following corollary, which is a half of the main theorem of Wang [14].

Corollary 5.4 ([14]). Every Clifford semigroup is Cayley-symmetric.

Let us conclude our discussion by the following example, which shows that in light of Theorem 5.3, we can construct non-regular, non-commutative but Cayley-symmetric semigroups.

Example 5.5. Let $Y=(\{\alpha, \beta, \gamma\}, \geq)$ be a semilattice where the partial order $\geq$ is defined by

$$
\geq=\{(\alpha, \alpha),(\beta, \beta),(\gamma, \gamma),(\alpha, \gamma),(\beta, \gamma)\}
$$

Let $S_{\alpha}, S_{\beta}$ be two dihedral groups. Let $S_{\gamma}=\{1\} \cup\{2 n \mid n \in \mathbb{Z}\}$. It is seen that $S_{\gamma}$ becomes a monoid with respect to the usual multiplication of integers. Define homomorphisms as follows:

$$
\begin{gathered}
\phi_{\alpha, \gamma}: S_{\alpha} \rightarrow S_{\gamma}, a \mapsto 1 \text { for all } a \in S_{\alpha}, \\
\phi_{\beta, \gamma}: S_{\beta} \rightarrow S_{\gamma}, b \mapsto 1 \text { for all } b \in S_{\beta}, \\
\phi_{\delta, \delta}=1_{S_{\delta}} \text { for all } \delta \in Y .
\end{gathered}
$$

Let $S$ be the strong semilattice of semigroups $\left\{S_{\delta}\right\}_{\delta \in Y}$, that is,

$$
S=\mathcal{S}\left[Y ; S_{\delta} ; \phi_{\delta, \lambda}\right] \text {. }
$$

It is clear that for each $\delta \in Y, S_{\delta}$ is not only self-decomposable but also Cayley-symmetric. By Theorem 5.3, $S$ is Cayley-symmetric. But note that the semigroup $S_{\gamma}$ is not regular. Furthermore, $S$ is also not regular. At last, $S$ is not commutative since nor is the dihedral group $S_{\alpha}$.

Acknowledgements. The author would like to thank Dr. Marcel Jackson for valuable comments and suggestions which lead to an improvement of this paper.

\section{References}

[1] Sr. Arworn, U. Knauer, and N. N. Chiangmai, Characterization of digraphs of right (left) zero unions of groups, Thai J. Math. 1 (2003), no. 1, 131-140.

[2] J. M. Howie, Fundamentals of Semigroup Theory, Clarendo Press, Oxford, 1995.

[3] A. V. Kelarev, On undirected Cayley graphs, Australas. J. Combin. 25 (2002), 73-78.

[4] _ Graph Algebras and Automata, Marcel Dekker, Inc., New York, 2003.

[5] - On Cayley graphs of inverse semigroups, Semigroup Forum 72 (2006), no. 3, 411-418.

[6] A. V. Kelarev and C. E. Praeger, On transitive Cayley graphs of groups and semigroups, European J. Combin. 24 (2003), no. 1, 59-72.

[7] A. V. Kelarev and S. J. Quinn, Directed graphs and combinatorial properties of semigroups, J. Algebra 251 (2002), no. 1, 16-26.

[8] - A combinatorial property and Cayley graphs of semigroups, Semigroup Forum 66 (2003), no. 1, 89-96.

[9] - A combinatorial property and power graphs of semigroups, Comment. Math. Univ. Carolin. 45 (2004), no. 1, 1-7. 
[10] A. V. Kelarev, J. Ryan, and J. Yearwood, Cayley graphs as classifiers for data mining: The influence of asymmetries, Discrete Math. 309 (2009), no. 17, 5360-5369.

[11] S. Panma, N. N. Chiangmai, U. Knauer, and S. Arworn, Characterizations of Clifford semigroup digraphs, Discrete Math. 306 (2006), no. 12, 1247-1252.

[12] S. Panma, U. Knauer, and S. Arworn, On transitive Cayley graphs of right (left) groups and of Clifford semigroups, Thai J. Math. 2 (2004), 183-195.

[13] - On transitive Cayley graphs of strong semilattices of right (left) groups, Discrete Math. 309 (2009), no. 17, 5393-5403.

[14] S. F. Wang, A problem on generalized Cayley graphs of semigroups, Semigroup Forum 86 (2013), no. 1, 221-223.

[15] R. J. Wilson, Introduction to Graph Theory, 3rd edn, Longman, New York, 1982.

[16] D. Yang and X. Gao, D-saturated property of the Cayley graphs of semigroups, Semigroup Forum 80 (2010), no. 1, 174-180.

[17] B. Zelinka, Graphs of semigroups, Casopis. Pest. Mat. 106 (1981), no. 4, 407-408.

[18] Y. Zhu, Generalized Cayley graphs of semigroups I, Semigroup Forum 84 (2012), no. 1, 131-143.

[19] _ Generalized Cayley graphs of semigroups II, Semigroup Forum 84 (2012), no. $1,144-156$.

[20] — On (n,m)-semigroups, Semigroup Forum 84 (2012), no. 2, 342-364.

School of Mathematics and Information Science

YANTAI UNIVERSITY

YAntai City 264005, P. R. ChinA

E-mail address: zyw@ytu.edu.cn 